\title{
Recursive approach to random sequential adsorption
}

\author{
D. J. Burridge ${ }^{1}$ and Y. Mao ${ }^{1,2}$ \\ ${ }^{1}$ Cavendish Laboratory, Madingley Road, Cambridge CB3 OHE, United Kingdom \\ ${ }^{2}$ Department of Physics and Astronomy, University of Nottingham, Nottingham NG7 2RD, United Kingdom
}

(Received 20 September 2003; published 24 March 2004)

\begin{abstract}
We present a generalization of Renyi's [A. Renyi, Trans. Math. Stat. Prob. 4, 205 (1963)] classical solution to the one-dimensional random sequential adsorption (RSA) problem, to the case where particle sizes are drawn from an arbitrary distribution, a process known as competitive RSA. We formulate the process using recursive relations, and without explicitly solving the equations, we extract limiting behavior which leads to exact analytic expressions for the final coverage. Our analysis is confirmed by computer simulations.
\end{abstract}

DOI: 10.1103/PhysRevE.69.037102

PACS number(s): 02.50.Ey, 05.20.Dd, 68.43.Mn, 81.10.Jt

The process of random sequential adsorption (RSA) models a diverse collection of physical phenomena, ranging from reactions on polymer chains [1] to protein deposition [2], and even ecological systems [3]. As a result, RSA has been the subject of intensive research [4-9] (see Ref. [9] for review).

The simplest example of one-dimensional (1D) RSA is the so called "car parking" problem: how many randomly parking motorists, on average, can be accommodated on a street of a given length? The 1D RSA problem has been solved analytically for the cases when all 'cars' are of equal length and "parking sites" are discrete $[1,10,11]$ or continuous [12]. Efforts have also been made to understand the RSA of multiple lengths, so called competitive random sequential adsorption (CRSA) [13-16]. These have yielded some limited analytical expressions for the final coverage due to a restricted class of adsorbant distributions [13,14], as well as numerical values for the coverage due to more general distributions [15]. However, no exact analytic results appear in the literature for the final coverage produced by a general distribution. Determining the time dependant behavior of the coverage is considerably more challenging [9]. Recently, an analytical solution has been found for the case of a binary mixture [17], but this relies on a carefully constructed casespecific ansatz, without suggesting a path to solutions of more general cases.

In this paper, we begin by reviewing Renyi's recursive formulation of the 1D RSA problem, where without explicitly solving the equations, the infinite length limit can be extracted (using Laplace transforms), leading to an exact analytic expression for the final coverage. We then generalize this exact solution to the CRSA of a mixture described by an arbitrary distribution function. This is our main result, from which we duly recover the analytical form for the saturation coverage due to a binary mixture, given by the infinite time limit of Ref. [17]. Finally, we present our computer simulations which confirm the analytical results.

Consider an empty length $x>1$, onto which blocks of unit length may be adsorbed. The first block can be randomly placed over an available length of $x-1$, after which its position is fixed, and any overlap of blocks is forbidden. Therefore, upon the first block being adsorbed, two independent gaps adding up to $x-1$ arise, see Fig. 1. The process of adsorption is sequential, such that two blocks cannot be adsorbed simultaneously. Let $f(x)$ be the number of blocks expected on average to eventually occupy an initially empty length $x$, then after the adsorption of the first block at $x^{\prime}$ the expectation becomes: $1+f\left(x^{\prime}\right)+f\left(x-1-x^{\prime}\right)$. Averaging over all possible initial sites, $x^{\prime}$, we have

$$
f(x)=1+\frac{1}{x-1} \int_{0}^{x-1} d x^{\prime}\left[f\left(x^{\prime}\right)+f\left(x-1-x^{\prime}\right)\right]
$$

which, thanks to its symmetry, can be simplified to

$$
f(x)=1+\frac{2}{x-1} \int_{0}^{x-1} d x^{\prime} f\left(x^{\prime}\right) .
$$

This is Renyi's master equation, which is analogous to the one first derived for the discrete case by Flory [1]. The initial condition for this integral equation is

$$
f(x)=0 \text { for } 0 \leqslant x<1
$$

because no blocks can be accommodated by a length less than 1 . Repeated substitution of $f(x)$ into the right-hand side of equation (2) leads to

$$
\begin{aligned}
f(x) & =1 \text { for } 1 \leqslant x<2 \\
& =\frac{3 x-5}{x-1} \text { for } 2 \leqslant x<3 \\
& =\frac{7 x-17-4 \ln (x-2)}{x-1} \text { for } 3 \leqslant x<4,
\end{aligned}
$$

and so on. The nested integration arising in the iterated form of Eq. (2) injects rapidly increasing number of terms, making a closed functional form for $f(x)$ rather impractical in contrast to the discrete case [1]. Numerically however, Eqs. (2) and (3) can be propagated to obtain the solution $f(x)$ for any $x$, providing a direct test of the asymptotic solution. For the more interesting case of CRSA this numerical solution has

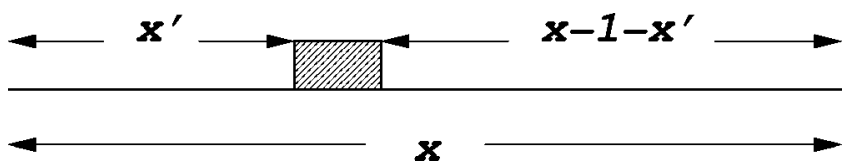

FIG. 1. Adsorption of the first block. 


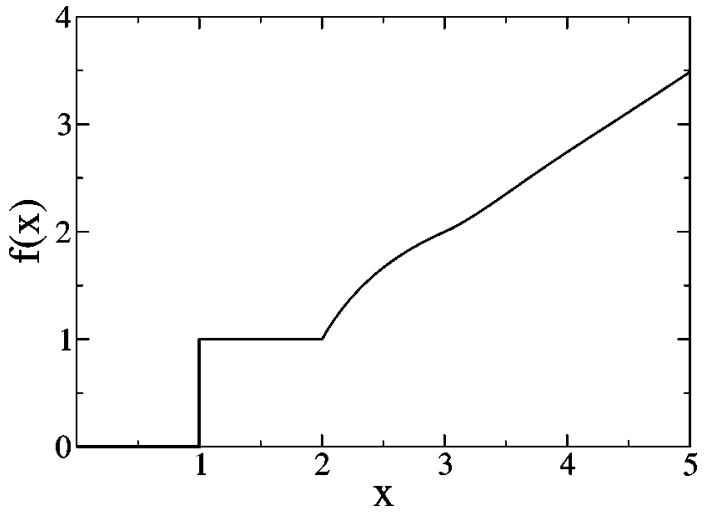

FIG. 2. The expectation of the number of blocks occupying an initially empty length $x$ at saturation, $f(x)$.

been used to test the saturation coverage of some specific distributions [15]. However, the numerical propagation for general distributions is rather cumbersome, and we turn to direct computer simulation. Figure 2 shows the function $f(x)$.

In the limit of large $x$, since the boundary effect decays over a finite length $[9,18]$ we expect the saturation coverage to increase linearly with $x$ :

$$
\lim _{x \rightarrow \infty} f(x)=\theta x
$$

and we are interested in this saturation coverage efficiency $\theta$, which can be extracted from the Laplace transform of $f(x)$, $F(p) \equiv \mathcal{L}[f]$, as the coefficient of the $p^{-2}$ term in the limit of $p \rightarrow 0$ :

$$
\lim _{p \rightarrow 0} F(p)=\frac{\theta}{p^{2}}
$$

Note that, only in the limit of $p \rightarrow 0$ is the Laplace integral dominated by large $x$ for which $f(x)=\theta x$ is justified.

It is now possible, without explicitly solving the master equation (2), to obtain the efficiency $\theta$. First differentiate the master equation (2) with respect to $x$, and then make the change of variables $x \rightarrow x+1$ so that the equation is valid for all $x>0$ :

$$
x f^{\prime}(x+1)=2 f(x)-f(x+1)+1 \text {. }
$$

The dash denotes differentiation with respect to the dependent variable. Taking the Laplace transform, we find that $F(p) \equiv \mathcal{L}[f]$ obeys

$$
F^{\prime}(p)+\left(1+\frac{2 e^{-p}}{p}\right) F(p)=-\frac{e^{-p}}{p^{2}},
$$

a linear first-order differential equation. By introducing the integrating factor

$$
\mu(p)=\exp \left[-\int_{p}^{k}\left(1+\frac{2 e^{-u}}{u}\right) d u\right]
$$

we can write the solution of Eq. (7) as

$$
F(p)=\mu(p)^{-1}\left[F(k)+\int_{p}^{k} \frac{\mu(w) e^{-w}}{w^{2}} d w\right],
$$

which is independent of the choice of $k$. We now make this solution explicit by taking $k$ to $\infty$ as follows. Since we know the initial form of $f(x)$ (see Fig. 1), we can deduce the functional form of $F(p)$ in the limit $p \rightarrow \infty$, where, knowing $f(x)=0$ on $[0,1)$ and $f(1)=1$ :

$$
\lim _{p \rightarrow \infty} F(p)=f(1) \int_{1}^{\infty} e^{-p x} d x=\frac{e^{-p}}{p} .
$$

This allows us to rewrite the solution, Eq. (9), in the form

$$
F(p)=\lim _{k \rightarrow \infty} \frac{e^{-p}}{p^{2}}\left[k g(p, k)+\int_{p}^{k} g(p, w) d w\right],
$$

with function $g$ defined as

$$
g(p, q)=\exp \left[-2 \int_{p}^{q} \frac{1-e^{-u}}{u} d u\right] .
$$

For consistency, it can be readily checked that the above expressions recover the limit (10) for $p \rightarrow \infty$. Now we consider $F(p)$ in the $p \rightarrow 0$ limit. Since

$$
\lim _{k \rightarrow \infty, p \rightarrow 0} k g(p, k) \propto \frac{1}{k} \rightarrow 0
$$

the first term in Eq. (11) may be dropped, which leads to

$$
\lim _{p \rightarrow 0} F(p)=\frac{1}{p^{2}} \int_{0}^{\infty} g(0, w) d w .
$$

The final coverage efficiency $\theta$ is the coefficient of $p^{-2}$, cf., Eq. (5):

$$
\theta=\int_{0}^{\infty} \exp \left[-2 \int_{0}^{w} \frac{1-e^{-u}}{u} d u\right] d w=0.7476 \ldots .
$$

This is the Renyi limit which may equivalently be obtained by numerical propagation of the Eqs. (2) and (3).

We now extend the analysis to the more general case of CRSA, where adsorbing blocks (of length $l$ ) are randomly drawn from a normalized distribution $\rho(l)$. Again we start with an empty length $x>l_{\max }$, where $l_{\max }\left(l_{\min }\right)$ are the largest (smallest) sized blocks in the distribution. The probability of a length $l$ block landing within $x$ is:

$$
P(l, x)=\frac{(x-l) \rho(l)}{x-\bar{l}},
$$

where average length is given by

$$
\bar{l}=\int_{0}^{\infty} l \rho(l) d l .
$$


The previous master equation (2) is then averaged over the landing probability $P(l, x)$ to obtain an equation for the average length covered, $f_{\rho}(x)$, given a distribution $\rho(l)$

$$
f_{\rho}(x)=\int_{0}^{\infty} d l P(l, x)\left[l+\frac{2}{x-l} \int_{0}^{x-l} f_{\rho}(y) d y\right]
$$

which is valid for all $x>l_{\max }$. The function $f_{\rho}(x)$ is the generalization of $f(x) . f_{\rho}(x)$ is still expected to increase linearly in the limit of large $x$ so that the Laplace analysis remains valid, cf., Eqs. (4) and (5). For the sake of simplicity and clarity, we shall present here the results for the restricted case of $l_{\max } \leqslant 2 l_{\min }$, e.g., Ref. [17]. In this case, for $x$ $<l_{\max }$, only a single adsorption event occurs before saturation, and the initial $f_{\rho}(x)$ is given by a limited average:

$$
f_{\rho}(x)=\frac{\int_{0}^{x} \rho(l) l(x-l) d l}{\int_{0}^{x} \rho(l)(x-l) d l} .
$$

The more general case of $l_{\max }>2 l_{\min }$ is straightforward in principle with calculations becoming more cumbersome as the ratio $l_{\max } / l_{\min }$ increases [19]. The CRSA equivalent of Eq. (7) is now given by

$$
F^{\prime}(p)+R(p) F(p)=U^{\prime}(p)+\bar{l} U(p)-S(p) e^{-p l_{\max },}
$$

where

$$
\begin{gathered}
R(p)=\bar{l}+\frac{2}{p} \int_{0}^{\infty} \rho(l) e^{-p l} d l, \\
U(p)=\int_{0}^{l_{\max }} f_{\rho}(x) e^{-p x} d x, \\
S(p)=\frac{\left(l_{\max }-\bar{l}\right) f_{\rho}\left(l_{\max }\right) p+\bar{l}}{p^{2}} .
\end{gathered}
$$

It is again possible to introduce an integrating factor and write down a solution analogous to Eq. (9). Noting that the first nonzero value of $f_{\rho}(x)$ is $f_{\rho}\left(l_{\text {min }}\right)=l_{\text {min }}$ then

$$
\lim _{p \rightarrow \infty} F(p)=\frac{e^{-p l_{\min }}}{p},
$$

which allows us to write the solution explicitly, and our limit analysis for $p \rightarrow 0$ leads to the final coverage, cf. Eq. (14):

$$
\begin{aligned}
\theta[\rho(l)]= & \int_{0}^{\infty} d w 2 w e^{\bar{l} w} \Phi(w) \int_{0}^{\infty} \rho(s) e^{-w s} d s \\
& \times \int_{0}^{\infty} \frac{\int_{0}^{y} \rho(l) l(y-l) d l}{\int_{0}^{y} \rho(l)(y-l) d l} e^{-w y} d y \\
& +\int_{0}^{\infty} d w e^{\left(\bar{l}-l_{\max }\right) w} \Phi(w) \\
& \times\left[w \int_{0}^{\infty} \rho(l) l\left(l_{\max }-l\right) d l+\bar{l}\right],
\end{aligned}
$$

where

$$
\Phi(w)=\exp \left[-2 \int_{0}^{w} \frac{1-\int_{0}^{\infty} \rho(l) e^{-u l} d l}{u} d u\right] .
$$

Equation (25) is our main result, which allows the determination of final coverage efficiency for adsorbing particles described by an arbitrary distribution function $\rho(l)$ provided $l_{\max }<2 l_{\min }$ (for $l_{\max }>2 l_{\min }$, the expression would be much more complicated). The infinite time limit predicted by the analytic work of Hassan et al. [17] for a binary mixture is a special case of our solution.

For the purpose of illustration, we take the adsorption of an equal binary mixture of lengths 1 and $m(m \leqslant 2)$ :

$$
\rho_{b}(l)=\frac{1}{2}[\delta(l-1)+\delta(l-m)],
$$

which leads to

$$
\theta(m)=\int_{0}^{\infty} h(m, w) G(0, w) d w,
$$

where

$$
G(p, w)=\exp \left(-\int_{p}^{w 2-e^{-u}-e^{-m u}} \frac{u}{u} d u\right)
$$

and

$$
h(m, w)=e^{[(m-3) / 2] w}+e^{[-(1+m) / 2] w}+\frac{m-1}{2} e^{[-(m-1) / 2] w} .
$$

Our analytic result, Eq. (28), agrees (after integration by parts) with the infinite time limit of the equal weight case of Hassan et al. [17]. Similarly, we can specify a top hat distribution between 1 and $m(m \leqslant 2)$ as

$$
\rho_{h}(l)=\frac{1}{m-1}[H(l-1)-H(l-m)]
$$

or a wedge function as

$$
\rho_{w}(l)=\frac{2}{m-1}[H(l-1)-H(l-m)](l-1),
$$




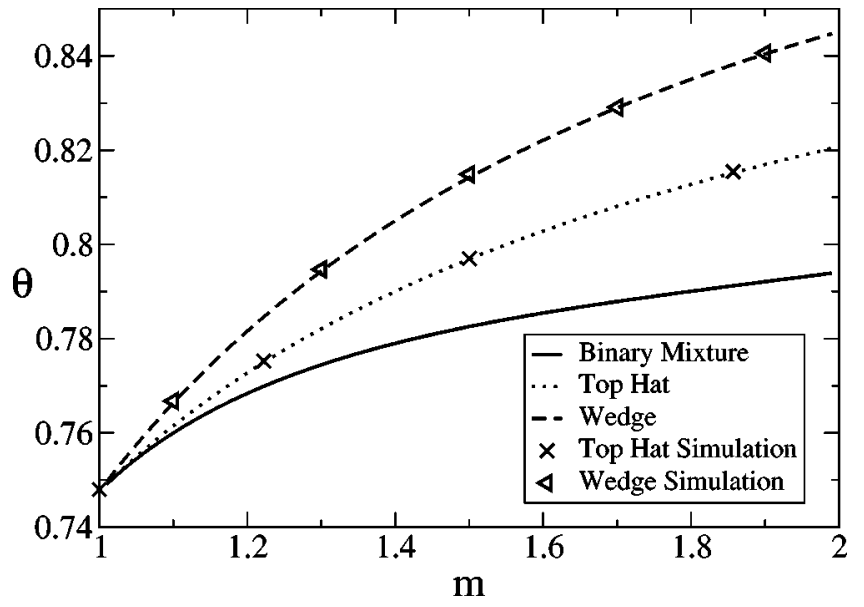

FIG. 3. The saturation coverage $\theta$ for three distributions where $l_{\text {min }}=1$ and $l_{\text {max }}=m$.

where $H(l)$ is the standard Heaviside step function. Substitution of these distribution functions into our Eq. (25) allows the final coverage to be computed within seconds (using standard software packages such as MATHEMATICA on a modern PC). The results are presented in Fig. 3, along with the results of computer simulations we carried out separately. The simulation points in Fig. 3 were obtained over days, and are in agreement with our analytic results to three significant figures.

It should be noted that this recursive technique may also be used to determine the distribution of gaps between neighboring blocks at saturation. Let $\gamma(x, l) d l$ be the expected number of gaps in the range $[l, l+d l]$, after saturating an initially empty length $x$ with unit length blocks, then we have

$$
\gamma(x, l)=\frac{2}{x-1} \int_{0}^{x-1} \gamma(y, l) d y .
$$

This equation is readily generalized to the case of a distribution of block lengths and solved using the same technique as described above [19].

In summary, we have generalized the recursive formulation of the 1D RSA problem to find an exact analytic expression for the final coverage produced by the CRSA of a general distribution of sizes. These analytic results have been confirmed by our computer simulations.

We would like to thank D. E. Khmelnitskii, P. D. Haynes, and R. Haydock for helpful discussions.
[1] P.J. Flory, J. Am. Chem. Soc. 61, 1518 (1939).

[2] J.J. Ramsden, Phys. Rev. Lett. 71, 295 (1993).

[3] N.T.J. Bailey, The Mathematical Theory of Infectious Diseases (Griffin, London, 1975).

[4] G.Y. Onoda and E.G. Liniger, Phys. Rev. A 33, 715 (1986).

[5] P. Schaaf and J. Talbot, Phys. Rev. Lett. 62, 175 (1989).

[6] J.W. Evans, Phys. Rev. Lett. 62, 2642 (1989).

[7] M.D. Grynberg and R.B. Stinchcombe, Phys. Rev. Lett. 74, 1242 (1995).

[8] N.V. Brilliantov, Y.A. Andrienko, P.L. Krapivsky, and J. Kurths, Phys. Rev. Lett. 76, 4058 (1996).

[9] J.W. Evans, Rev. Mod. Phys. 65, 1281 (1993); J. Talbot, G. Tarjus, P.R. van Tassel, and P. Viot, Colloids Surf., A 165, 287
(2000).

[10] E.A. Boucher, Trans. Faraday Soc. 69, 1839 (1973).

[11] I.R. Epstein, Biopolymers 18, 765 (1979); 18, 2037 (1979).

[12] A. Renyi, Trans. Math. Stat. Prob. 4, 205 (1963).

[13] P.E. Ney, Ann. Math. Stat. 33, 702 (1962).

[14] J.P. Mullooly, J. Appl. Probab. 5, 427 (1968).

[15] A.S. Goldman, H.D. Lewis, and W.M. Visscher, Technometrics 16, 301 (1974).

[16] B. Mellein and E.E. Mola, J. Math. Phys. 26, 514 (1985).

[17] M.K. Hassan, J. Schmidt, B. Blasius, and J. Kurths, Phys. Rev. E 65, 045103 (2002).

[18] E.S. Page, J. R. Stat. Soc. Ser. B. Methodol. 21, 364 (1959).

[19] D.J. Burridge and Y. Mao (unpublished). 